\title{
A case of penetrating buttock trauma and lessons learnt on how new protocols of the Northern Ireland Major Trauma Network can improve patient care
}

\author{
Dimitrios Angelou ${ }^{1}$, Mark McKeever ${ }^{1}$, Amy Doran ${ }^{1}$, and Momin Malik ${ }^{1}$ \\ ${ }^{1}$ Affiliation not available
}

November 23, 2021

\begin{abstract}
We are reporting a case of a patient who was brought to our emergency department after having sustained a penetrating buttock trauma and we are discussing about the importance of good Inter-hospital collaboration to provide patients with early access to Major Trauma Centres with a specific focus on Northern Ireland protocols.

Our patient is a 54-year-old lady who fell from a stepladder onto the metallic base of an artificial Christmas tree causing the stem of the tree to impale her through her right buttock into her pelvis.

Despite the impressive mechanism of injury, there was no immediate life-threatening bleed and the patient was subsequently transferred to the local Major Trauma Centre for expert surgical management but suffered significant delays due to suboptimal communication and lack of clear transfer pathways.

Current literature recognises that penetrating gluteal trauma should be considered a life-threatening injury and this case highlights learning points around the management of penetrating trauma in terms of investigations, early transfer and definitive surgical treatment.

We discuss about the initial management, imaging and surgical considerations for this case.

We also discuss about the current recommendations for early transfer between smaller centres to Major Trauma Centres and the Northern Ireland specific protocols that have been introduced by the Northern Ireland Major Trauma Network. We focus on the Call and Send protocol and on the NIMTN Major Trauma Triage Tool.
\end{abstract}

\section{INTRODUCTION - TRAUMA PROTOCOLS FOR NORTHERN IRELAND}

The Northern Ireland Major Trauma Network (NIMTN) has created regional protocols in order to guide practice and coordinate trauma services between the different HSC trusts in Northern Ireland. [1]

The NIMTN is active on the planning of every aspect of trauma care ranging from the pre-hospital care to the rehabilitation care but we are only going to focus on the emergency transfer protocols for major trauma. A basic principle of the guidance is that the patient should be receiving the right care in the right place at the right time.

Northern Ireland's Major Trauma Centre is the Royal Victoria Hospital in Belfast and is receiving patients from all other local hospitals in the region. 
The relevant protocols in action are the Call and Send Protocol and also the Major Trauma Triage Tool. They aim to define the criteria for direct transfer to a Major trauma centre versus the nearest district hospital and also provide a clear guidance on the process for a transfer of major trauma to the major trauma centre in order to avoid unnecessary delays.

The call and send protocol has been in use since January of 2021 and is an extension of the major trauma triage tool. It uses the injury severity score and facilitates transfer from district general hospitals to the major trauma centre. To be exact, patients with calculated injury severity score more than 15 qualify for expediated transfer to the MTC. That indicates that the patient has at least a single system severe injury or multiple system minor/ moderate injuries. The Local hospital senior ED doctor contacts the MTC where the patient cannot be refused and a hyperacute transfer is arranged.

This protocol reduces the clinical debate of accepting the patient transfer and it provides a direct trauma line for efficient communication.

The Major Trauma Triage Tool aims to direct all major trauma to the MTC to avoid unnecessary delays. Decisions are made against set of criteria for severe injuries including: 1. Vital signs and level of consciousness, 2. high risk anatomy of the injury, 3. mechanism of injury.

If the severity criteria are fulfilled and the estimated arrival time to the major trauma centre is less than 45 minutes then the patient is transferred directly to the MTC.

Further information and the exact protocols can be found on the NIMTN website http://www.hscboard.hscni.net/majortrauma/nimtn-contacts/.

Penetrating buttock injuries are rare but potentially life-threatening injuries [2] and this case highlights learning points around the early management of penetrating buttock trauma, the current transfer protocols and the specific surgical considerations. This report aims to aid doctors consolidate their knowledge around the subject and improve their management of similar situations.

We also aim to raise awareness regarding the Northern Ireland Major Trauma Network transfer protocols that were not available at the time of the event.

\section{CASE PRESENTATION}

A 54-year-old lady, with no significant pre-existing medical comorbidities, was brought in by ambulance to the emergency department of our district general hospital after falling from a stepladder onto the metallic base/ stem of a half dismantled artificial Christmas tree while removing Christmas decorations in her home.

She arrived lying prone, with approximately $50 \mathrm{~cm}$ of the metallic stem and base-plate (diameter approximately $60 \mathrm{~cm}$ ) protruding inferiorly and laterally from her right buttock, near the gluteal fold. She was conscious, communicating normally and haemodynamically stable. Intravenous access had been established by the ambulance crew, and opiate analgesia given. She had her arms around a pillow, and did not have a cervical collar on. There was a small amount of congealed blood around the entry wound. A careful abdominal and perineal examination was performed, including per rectum and per vagina digital exams. Accepting the limitations due to the patient's prone position, and the desire not to move or dislodge the foreign body, all examinations were unremarkable.

\section{INVESTIGATIONS}

Trauma X-rays in the emergency department included a pelvic X-ray (Figure 1), which showed the radiopaque metal pipe's path from the right buttock entry point, traversing obliquely through the pelvis and lower abdomen, with the tip lying in the left upper abdominal quadrant. No bony injury or free air was identified.

In view of the position of the foreign object, the pelvic and abdominal neurovascular structures, rectum, urinary bladder, uterus, vagina and bowel were all considered to be at risk of injury. Removal of the 
object in theatre under general anaesthesia was considered the safe option, with colorectal, gynaecological, orthopaedic, vascular and interventional radiological expertise on standby in case needed.

The case was discussed with the regional Major Trauma Centre for immediate transfer. Their advice, however, was to perform a Computerised Tomography (CT) scan locally prior to transfer, in order to fully assess the extent of the injury. The extracorporeal length of the metal pipe and base plate were unable to fit through the donut of the CT scanner. Sawing off the base plate was considered risky in case the movement exacerbated any internal injuries. Assistance was sought from the local fire department, who used metal cutters to snap off and shorten the stem to a manageable length.

The CT scan (3D reconstructed images Figures 2 and 3) demonstrated the foreign body penetrating the right buttock close to the sciatic nerve, traversing through the sciatic foramen, crossing the midline in contact with the sigmoid colon, loops of small bowel within the pelvis and the left common iliac artery. It terminated in the left upper abdomen adjacent to multiple small bowel loops. A small volume haematoma within the pelvis was also noted but no free air or other abdominal visceral injuries were seen.

The above investigations required a significant amount of time including one and a half hour for fire brigade to cut off the metal pole for the patient to fit in the CT scanner, forty minutes for the CT scan and report and two and a half hours of debating for trauma specialities to accept the transfer. The total delay of transfer was three hours and forty minutes.

\section{TREATMENT AND OUTCOMES}

The patient remained clinically stable throughout, and was transferred to the local MTC for definitive management seven hours after presentation. The foreign body was removed under direct vision in theatres, during a laparotomy by the vascular surgeons. No major bleeding or visceral damage was identified and the entry wound was closed primarily. The patient had an uneventful recovery and was discharged home after four days. One- and six-month post-operative out-patient clinic visits raised no further issues.

\section{DISCUSSION}

Penetrating buttock trauma is accounts for $2-3 \%$ of penetrating injuries to the torso; with stab wounds, gunshots and transfixation injuries being more common than impalement. The mechanisms of injury for impalement injuries include falls from a height onto a protruding hard object [3,4] and high-speed collisions [5]. They can be potentially life threatening due to the risk of injury to a number of critical abdominal and pelvic vessels, with a mortality of $4 \%-11 \%[6,7]$. The severity of the injury should not be underestimated even if it initially appears innocuous or the entry would small [8].

Initial Management should follow ATLS guidelines. During the initial assessment the foreign body must not be removed from the injury site [4] as further bleeding may be caused from the removal process. Early life saving interventions like major haemorrhage protocol activation, radiologic embolization or damage control surgery may be needed [6].

In stable patients, the imaging modality of choice to assess visceral injuries is CT [4,6,9]. Oral, intravenous and rectal contrast as well as endoscopy may be used. Visceral and vascular injuries are common, the frequency of various visceral and vascular injuries reported in literature are shown in Table 1.

While a selected group of patients with penetrating gluteal injuries may be managed conservatively [6], surgery remains a cornerstone in the management. Surgery is essential in the presence of haemodynamic instability, hemoperitoneum or injury to major vessels, full thickness injury to bowel, peritonitis and perforation of other hollow viscus organs including the urinary bladder. Surgical exploration can be challenging due to the potentially lengthy intra corporeal tract, and the number and nature of viscera involved. An endoscopic alternative to open exploration, using the spinal endoscope, has been described for use in relatively uncomplicated cases [10]. Angioembolisation is another invaluable tool, either alone or in tandem with surgery, especially in haemodynamically unstable patients or for failed control of bleeding after surgery. It 
is particularly helpful in dealing with injuries to branches of the internal iliac artery, superior gluteal artery, and for the treatment of pseudoaneurysms [6].

Better survival is reported when the patient is transferred early to a trauma unit or equivalent [4], as there may be need for multi-speciality approach; including vascular surgery [6] and interventional radiology [6]. Transfer should never be delayed to carry out investigations which will not affect the transfer decision. A number of tools, such as the Injury Severity Score, have been formulated to aid in decision-making (NICE guidelines NG40). Rapid transfer protocols should be in place in order to facilitate timely transfer to the trauma centre with a target time for leaving the unit in less than 30 minutes from arrival [11]. Despite this, inappropriate delays to transfer are frequently reported in literature [12]. This is especially critical in areas like Northern Ireland and Scotland, where the population is dispersed, and journey times can be affected by weather and road conditions.

In our case there were significant delays related to investigations and transfer which is against the current guidelines. The patient was haemodynamically stable but had a severe enough injury that put them at risk of acute deterioration due to bleeding. For that reason, imaging was performed prior to transport.

\section{CONCLUSIONS}

* Penetrating buttock trauma is a potentially life-threatening condition and should be treated as such according to local major trauma protocols.

* Initial management should be according to ATLS guidelines. Do not remove the foreign body without ensuring availability of resources to deal with complex abdominal or pelvic haemorrhage.

* Early transfer to a trauma centre is a key management consideration. Do not delay transfer for the sake of investigations which will not impact immediate management or transfer.

* Surgical intervention is a cornerstone for the management of impalement injuries.

* Northern Ireland specific guidance for major trauma transfer include the Call and Send Protocol and also the Major Trauma Triage Tool to reduce unnecessary delays in transfer.

\section{FIGURES}

\section{Figure 1}

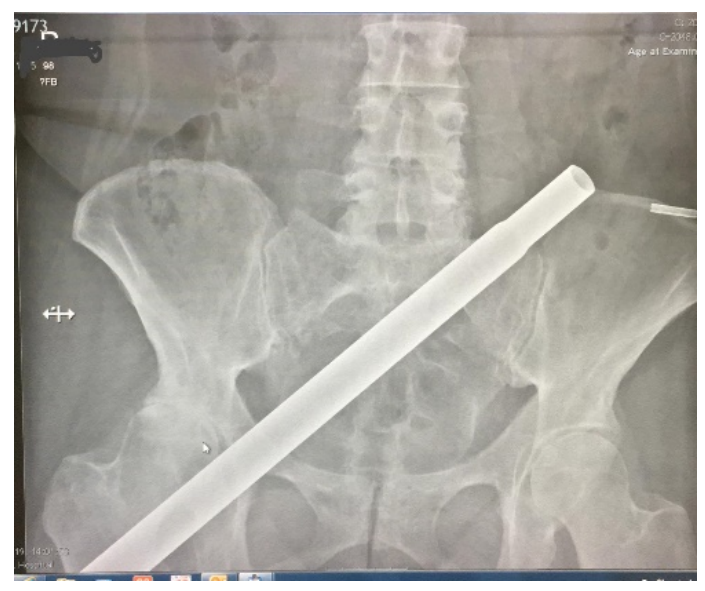




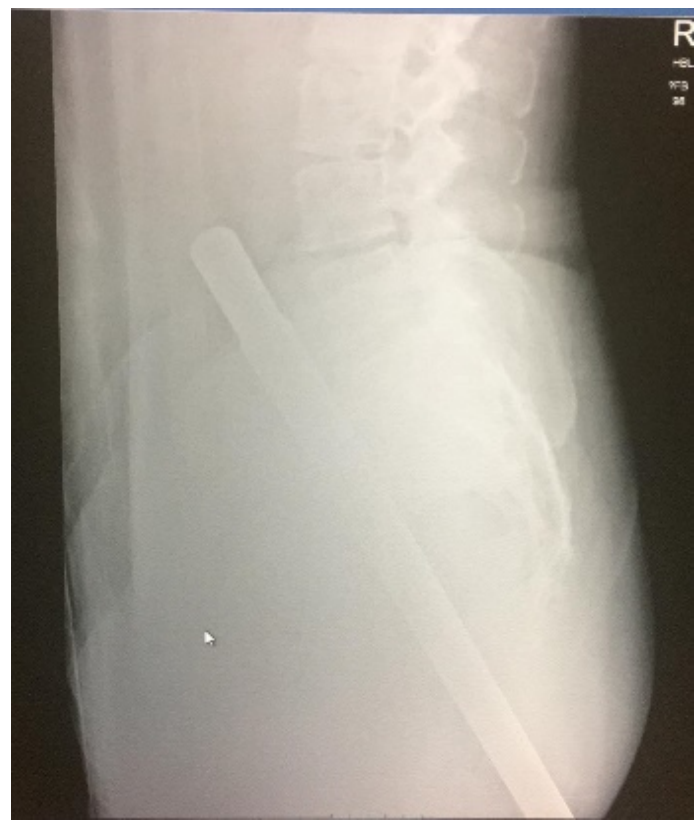

Figure 1 - an x ray showing the Christmas tree base penetrating through the abdomen Figure 2

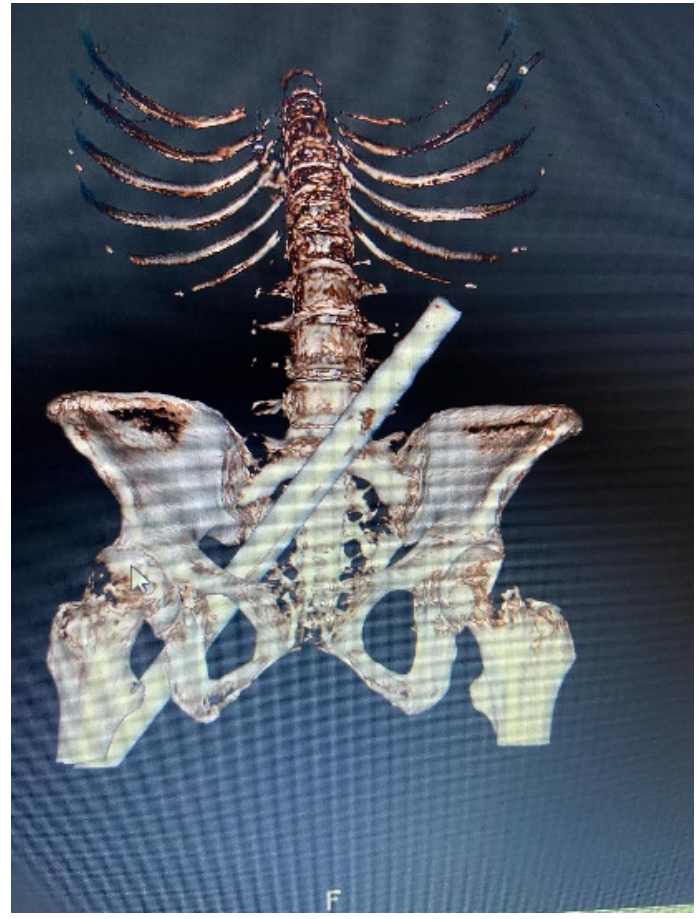



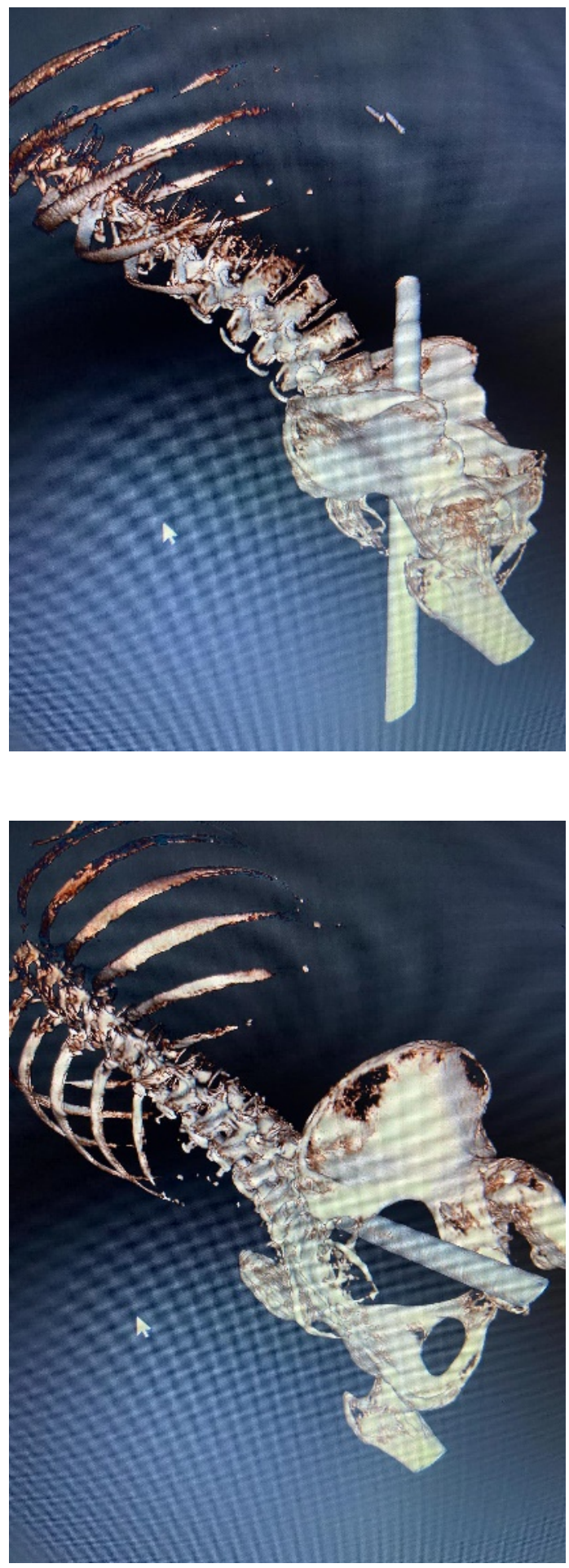

Figure 2 - 3D reconstructed CT scan Images demonstrating the path of the foreign body Figure 3 


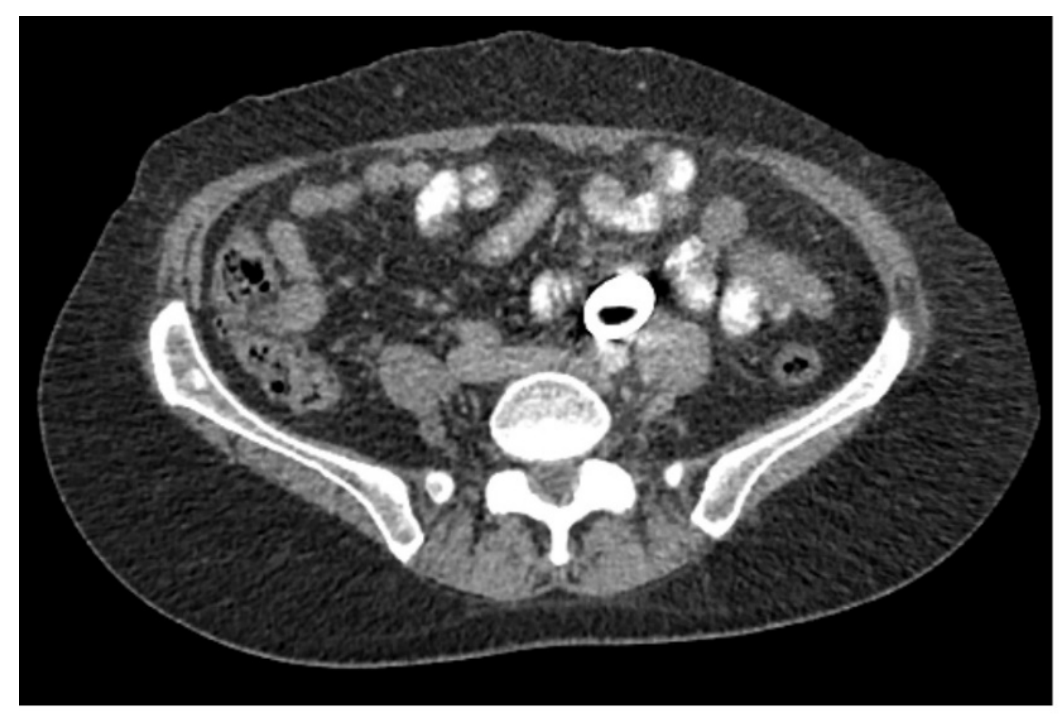

Figure 3- CT scan image demonstrating the relations of the foreign body to intra-abdominal structures

Table 1. Frequency of Reported Major Injuries in 615 Patients

Adapted from: (Figure 1. Types of major injury in 615 patients with penetrating trauma to the buttock.) Lunevicius R, Schulte K-M. Analytical review of 664 cases of penetrating buttock trauma. World J Emerg Surg [Internet]. 2011;6(2):33. Available from: http://www.wjes.org/content/6/1/33

\begin{tabular}{llll}
\hline Small Bowel & $8.3 \%$ & Inferior Gluteal Artery & $0.7 \%$ \\
\hline Colon & $6.3 \%$ & Testis & $0.5 \%$ \\
Superior Gluteal Artery & $5.4 \%$ & Hypogastric Artery & $0.5 \%$ \\
Rectum & $4.9 \%$ & Femoral Vein & $0.5 \%$ \\
Bony Pelvis & $4.4 \%$ & Prostate & $0.3 \%$ \\
Urinary Bladder & $3.7 \%$ & Liver & $0.3 \%$ \\
Iliac Artery & $2 \%$ & Internal Pudendal Artery & $0.3 \%$ \\
Major Vessel & $1.6 \%$ & Ureter & $0.2 \%$ \\
Urethra & $1.5 \%$ & Ovaries & $0.2 \%$ \\
Corpora Cavernosa/ Scrotum & $1 \%$ & Iliac Vessel & $0.2 \%$ \\
Retroperitoneal Hematoma & $1 \%$ & Sacroiliac Joint capsule & $0.2 \%$ \\
Sciatic Nerve & $0.8 \%$ & Sacrospinous Ligament & $0.2 \%$ \\
Femoral Artery & $0.8 \%$ & Popliteal Artery & $0.2 \%$ \\
Iliac Vein & $0.8 \%$ & Inferior Vena Cava & $0.2 \%$ \\
Lumbosacral Plexus & $0.7 \%$ & Superior Mesenteric Artery & $0.2 \%$ \\
\hline
\end{tabular}




\section{PATIENT'S PERSPECTIVE}

The patient kindly replied to us with her feedback in writing:

"Just want to add that I received excellent care and attention from ambulance to hospital. All staff at hospital were extremely kind and supportive and simply owe my life toto them.

The aftercare from my local community was incredible also.

The whole accident was a very worrying time for my family but fortunately I've recovered fully.

Thank you so much"

\section{REFERENCES}

1. D Redmill, E Giddings. Call \& Send Protocol for Major Trauma. Northern Ireland Major Trauma Network Standard Operating procedure. January 2021.

2. Lunevicius R, Schulte K-M. Analytical review of 664 cases of penetrating buttock trauma. World J Emerg Surg [Internet]. 2011;6(1):33. Available from: http://www.wjes.org/content/6/1/33

3. Oya S, Miyata K, Yuasa N, Takeuchi E, Goto Y, Miyake H, et al. Impalement injury to the left buttock with massive bleeding: A case report. Nagoya J Med Sci. 2014;76(1-2):147-52.

4. Ugoletti L, Zizzo M, Castro Ruiz C, Pavesi E, Biolchini F, Annessi V. Gluteal, abdominal, and thoracic multiple impalement injuries: A case report on management of a complex polytrauma. Medicine (Baltimore). 2019;98(22):e15824.

5. Weymann A, Matter P. [Penetrating injury]. Zeitschrift fur Unfallchirurgie und Versicherungsmedizin Off Organ der Schweizerischen Gesellschaft fur Unfallmedizin und Berufskrankheiten = Rev Traumatol d'assicurologie organe Off la Soc suisse . 1993;Suppl 1:247-51.

6. Lunevicius R, Lewis D, Ward RG, Chang A, Samalavicius NE, Schulte KM. Penetrating injury to the buttock: an update. Tech Coloproctol. 2014;18(11):981-92.

7. Ceyran H, Akçali Y, Özcan N, Tasdemir K. Isolated penetrating gluteal injuries. Perspect Vasc Surg Endovasc Ther. 2009;21(4):253-6.

8. Hefny AF, Salim EA, Bashir MO, Abu-Zidan FM. An unusual stab wound to the buttock. J Emergencies, Trauma Shock. 2013;6(4):298-300.

9. Heller MT, Oto A, Allen BC, Akin O, Alexander LF, Chong J, et al. ACR Appropriateness Criteriaß Penetrating Trauma-Lower Abdomen and Pelvis. J Am Coll Radiol [Internet]. 2019;16(11):S392-8. Available from: https://doi.org/10.1016/j.jacr.2019.05.023

10. Long ZS, Nie XY, Zhang YW. Treatment of penetrating trauma to the buttock assisted by spinal endoscopy. J Int Med Res. 2019;48(1).

11. National Clinical Guideline Centre. Major Trauma: Service Delivery. Natl Inst Heal Care Excell [Internet]. 2020;(February):1-8. Available from: http://www.ncbi.nlm.nih.gov/pubmed/26913313

12. Harrington DT, Connolly M, Biffl WL, Majercik SD, Cioffi WG, Britt LD, et al. Transfer times to definitive care facilities are too long: A consequence of an immature trauma system. Ann Surg. 2005;241(6):961-8. 